\title{
The future of genetics in everyday medicine
}

\author{
N Thalayasingam \\ Specialty Registrar, Department of Clinical Genetics, Western General Hospital, Edinburgh, UK
}

\begin{abstract}
Genetics is a developing field that increasingly influences medical practice in a large number of specialties. This symposium highlighted how current research is providing insights into the genetic causes of disease, leading to a deeper understanding of the pathophysiology and identification of potential novel treatment strategies. We were provided with an overview of developments in monogenic conditions such as Marfan's syndrome and Duchenne muscular dystrophy and complex traits such as atopic dermatitis. The practicalities of providing genetic testing for more common conditions such as familial hypercholesterolaemia were discussed, highlighting the more prominent role clinical genetics may come to play in mainstream medicine.
\end{abstract}

Published online June 2009

Correspondence to $\mathbf{N}$ Thalayasingam, Department of Clinical Genetics, Western General Hospital, Crewe Road South, Edinburgh EH4 2XU, UK

tel. $+44(0) / 315371095$

e-mail nishanthi_t@hotmail.com

KEYWORDS Atopic dermatitis, Duchenne muscular dystrophy, familial hypercholesterolaemia, genetics, Marfan's syndrome, obesity

DECLARATION OF INTERESTS No conflict of interests declared.

Clinical genetics has traditionally been considered to be a diagnostic specialty, but we may be reaching the stage when genetics begins to inform treatment, starting a new era of translational medicine.

\section{SESSIONS I \& 2 FROM MOLECULES TO TREATMENT}

Professor Julian Sampson (Medical Genetics, University of Cardiff) provided an overview of the research carried out by his team and others on tuberous sclerosis (TS). This autosomal dominant condition is characterised by a neurodermatosis with hamartoma formation and significant neuropsychiatric features. Up to a third of patients meet the diagnostic criteria for autism, the strongest link between any Mendelian disorder and autism.

Work over the past 20 years has led to the identification of the two genes mutated in this condition, TSCI and TSC2. The protein products of TSCI and TSC2 exist as a complex which inhibits mTOR, a protein kinase central to the regulation of cell proliferation and metabolism.' Understanding the molecular basis of this disease has helped to identify possible novel treatment options by the inhibition of the mTOR pathway. Rapamycin (sirolimus), a known mTOR inhibitor, could potentially replace the loss of TSCI and TSC2 activity. The first clinical studies showed a dose-related response, with shrinkage of the renal tumours that was measurable by magnetic resonance imaging (MRI). ${ }^{2}$ However, the effect was reversible.

Tumours are only one aspect of TS and it is the neuropsychiatric features, such as seizures and behavioural problems, which cause the most distress to parents. This area is more difficult to study, but mouse models of TS have shown improvements in spatial learning following treatment with rapamycin. ${ }^{3}$
Aortic aneurysms account for $1-2 \%$ of deaths in the Western world. It is reported that $20 \%$ of thoracic aortic aneurysms are familial, with a high degree of genetic heterogeneity. Professor Bart Loeys (Centre for Medical Genetics, Ghent, Belgium) described how work on syndromic causes of aortic aneurysms has aided our understanding of the underlying pathogenesis.

Marfan's syndrome has been used as a paradigm to study aneurysmal disease.The early hypothesis that the causative mutations in fibrillin I led to microfibrillar deficiency and structural changes in the vascular wall did not explain the additional features of the condition. New evidence from mouse research has shown that the multisystem effects may originate in changes in transforming growth factor $\beta$ (TGF- $\beta$ ) signalling, rather than primarily a structural protein change. ${ }^{4}$ This has been reinforced by the identification of a new aortic aneurysm syndrome, LoeysDietz syndrome, where affected families have been shown to have mutations in genes encoding TGF- $\beta$ receptors. ${ }^{5}$

Losartan, an angiotensin II receptor antagonist known to have an inhibitory effect on TGF- $\beta$, reduces the rate of aortic root growth in mouse models of Marfan's syndrome, compared with placebo and propanolol. ${ }^{6}$ This was independent of the haemodynamic effects of the drugs. Early clinical trials have been encouraging, with losartan appearing to target the areas of abnormal growth, and large prospective randomised trials are currently under way. ${ }^{7}$ If TGF- $\beta$ signalling is part of the final common pathway of aneurysm formation this may allow preventative therapy, rather than symptomatic treatment, in select groups of patients.

Dr Elizabeth Patton (MRC Human Genetics Unit, Edinburgh) provided an insight into her research in melanoma formation using the zebrafish as an ideal 
model organism. Melanomas are notoriously difficult to treat and the hope is that clues may come from understanding the development of the normal melanocyte, as these pathways are misregulated in melanoma. Indeed, such work has shown that melanocytes are derived from the highly motile neural crest and have enhanced anti-apoptotic capabilities, making them naturally resistant to cytotoxic agents.

The Cancer Genome Project identified the role of BRAF mutations in melanoma leading to the development of zebrafish models of human melanoma. ${ }^{8}$ Somatic mutations in BRAF can result in the development of the common mole and play a role in melanoma progression. ${ }^{9}$ Models allow the observation of melanocyte development, movement and death in this setting and in response to the addition of small molecules. This work may identify potential therapeutic targets.

Atopic dermatitis is a common, complex disease with a number of strong genetic predisposing factors. Professor Irwin McLean (University of Dundee) described how work by his team following the monogenic condition ichthyosis vulgaris has led to insights into the aetiology and possible treatment options for this common condition.

Ichthyosis vulgaris (common dry, flaky skin), due to mutations in the filaggrin gene, is the most common monogenic skin disorder, affecting one in 90 of the British population. ${ }^{10}$ Filaggrin is important for the structure of the stratum corneum and is a natural moisturiser. A deficiency results in the disruption of the skin barrier and the possible entry of usually unseen pathogens."

The links to atopic dermatitis came from the clinical recognition of the high level of eczema in families with ichthyosis vulgaris combined with the genetic evidence that the filaggrin gene was located in an atopic dermatitis susceptibility locus. Up to I $4 \%$ of the British population are heterozygous carriers for a null mutation in filaggrin, having $50 \%$ of the normal amount of filaggrin and subclinical, dry skin. However, they are at high risk of atopic dermatitis and its related conditions: atopic asthma, allergic rhinitis and food allergies. Large population studies have shown $40 \%$ penetrance of eczema in this group, high for a complex trait. ${ }^{12}$ The $1 \%$ of the population homozygous for filaggrin mutations have the overt phenotype of ichthyosis vulgaris and are almost $100 \%$ likely to develop atopic dermatitis or one of the other secondary allergic characteristics.

The analysis of a cohort of Dundee children demonstrated that filaggrin mutations are a risk factor for asthma. However, this risk was only for the children with atopic dermatitis, suggesting that there are at least two types of asthma; one driven by skin barrier defects and a second with a differing, unknown aetiology.
The role of the changes in the skin barrier has led to a review of the previously widely held hypothesis that atopic dermatitis is primarily due to a heightened $\mathrm{T}$ helper cell 2 (Th2) immune response, as this now appears to be downstream of the primary defect. Professor McLean's team is currently looking at ways to increase filaggrin expression in mutation carriers.

The Sydney Watson Smith Lecture was given by Professor Gert-Jan van Ommen (Leiden University Medical Centre, the Netherlands), who described an exciting strategy for the treatment of Duchenne muscular dystrophy (DMD), providing an insight into the challenges of clinical research in young patients. Duchenne muscular dystrophy and Becker muscular dystrophy (BMD) are neuromuscular conditions caused by changes in the dystrophin gene. However, while DMD is a severe condition with childhood onset, BMD is late onset and considerably milder. The clinical difference stems from the type of dystrophin gene defect. In DMD the reading frame of the gene is disrupted, producing a prematurely terminated, non-functioning protein. By contrast, BMD mutations do not alter the reading frame and so generate an internally deleted but partly functional dystrophin protein.

This distinction led to the hypothesis that correcting the reading frame in DMD could allow the production of a shorter yet still functional protein and so improve the clinical picture to that of BMD. Antisense oligonucleotides (AONs) effectively target the exons to be skipped, allowing correction of the reading frame downstream of the mutation and resulting in a shortened but active dystrophin protein.

Initial clinical trials in four DMD patients where AONs were injected into the tibialis anterior and dystrophin levels assessed on muscle biopsies were remarkably encouraging. ${ }^{13}$ Significant work remains to optimise dosing, delivery systems and the identification of suitable biomarkers to monitor disease. Early studies have focused on AONs to exon $5 \mathrm{I}$ defects, which represent around $13 \%$ of DMD patients. This is truly personalised therapy, as the choice of $A O N$ is determined by the individual's gene defect, but this new field has implications for people with rarer mutations.

\section{SESSION 3 \\ FAMILIAL HYPERCHOLESTEROLAEMIA}

The third session of the day focused on familial hypercholesterolaemia $(\mathrm{FH})$ and the identification of individuals at risk. Professor Steve Humphries (Chief Executive Officer, UCL Genetics Institute, London) provided an overview of this underdiagnosed, treatable, autosomal dominant condition. ${ }^{14}$ Surveys from UK lipid clinics have shown that we know of approximately 15,000 cases but, with a prevalence of one in 500 , this leaves more than $85 \%$ of individuals undiagnosed. 
In $\mathrm{FH}$, high levels of low-density lipoprotein cholesterol (LDL-C) are present from birth. The analysis of patients on the Simon Broome Register in the pre-statin era demonstrated a greater than 23-year reduction in life expectancy in comparison with the general population. In approximately $80 \%$ of clinically diagnosed patients a genetic cause can be identified.

Professor Humphries advocated cascade screening, which has been used in this setting in the Netherlands. ${ }^{15}$ The lipidologist plays a central role and an $\mathrm{FH}$ nurse leads cascade screening using cholesterol and DNA testing. This has been shown to be acceptable and viable in terms of cost per life years gained. ${ }^{16}$

In contrast, Dr David Wald (Senior Lecturer and Consultant Cardiologist, Wolfson Institute for Preventive Medicine, London) proposed a population screening strategy. Analysis of the distribution of total cholesterol at various ages shows a marked overlap in measured cholesterol levels in $\mathrm{FH}$ and non-FH individuals. ${ }^{17}$ Cholesterol levels offer maximum discrimination for screening in the one to nine years age group, where the overlap is lowest. Heel prick cholesterol screening at the time of childhood immunisations was proposed. ${ }^{18}$ However, given the prevalence is one in 500 and the false positive rate one in 1,000 , this raises the question of whether we would mislabel a relatively large number of children.

The recent National Institute for Health and Clinical Excellence (NICE) guidelines recommended a nationwide, family-based follow-up system to optimise cascade screening. ${ }^{14}$ Dr Michael Wright (Clinical Director, Northern Genetics Service, Newcastle) proposed that, as this describes the system long established in clinical genetics, this specialty should lead screening, ideally through a genetic counsellor linked to the lipid clinic.

Familial hypercholesterolaemia may mark a turning point in clinical genetics as it highlights the need to consider how to deliver genetics care and counselling in mainstream medicine.

\section{SESSION 4 BEING OVERSIZED}

Professor Eamonn Maher (University of Birmingham) discussed the process of epigenetic modification, whereby expression of specific genes is altered without changes in the DNA sequence. One example is genomic imprinting, where expression of a gene differs between the maternally and paternally derived alleles due to methylation differences. Imprinting affects up to $0.5 \%$ of genes but is disproportionately represented in disorders associated with growth.

Constitutional changes in imprinting can lead to the rare congenital overgrowth syndrome Beckwith-Wiedemann syndrome (BWS). Somatic changes in the imprinting of genes involved in BWS, such as IGF2, are implicated in human cancers such as Wilm's tumour and colorectal cancers. ${ }^{19}$ More recently, it has been shown that the environment can have a direct impact on genes, as assisted reproduction technology (ART) has been associated with an increase in disorders of genomic imprinting. ${ }^{20}$

We know that recent increases in the prevalence of obesity are driven by environmental changes but, despite such changes, we are not all affected. Professor Steve O'Rahilly (Department of Clinical Biochemistry, University of Cambridge) described evidence that, despite environmental changes, around $70 \%$ of our body mass index (BMI) remains heritable. ${ }^{21}$

There is considerable interest in the elucidation of genetic factors that influence our weight and their mechanism of action. Loci associated with obesity, identified through genome-wide association studies, contain genes highly expressed or known to act in the central nervous system, implicating the role of the central nervous system in the predisposition to obesity. ${ }^{22}$

A common variant at the FTO (fat mass and obesity associated) locus has been convincingly shown to influence $\mathrm{BMI}$ and, for the $16 \%$ of people homozygous for this risk allele, $3 \mathrm{~kg}$ is added to their weight. ${ }^{23}$ The $40 \%$ of people heterozygous for the allele add I.5 kg to their weight. An analysis of appetite ratings indicated increased spontanteous food intake and decreased satiety. ${ }^{24}$ Interestingly, such individuals have a higher than expected energy expenditure, hence their not being considerably more overweight.

\section{CONCLUSION}

The symposium provided an exciting overview of current research in genetics. This information furthers our understanding of human development and disease mechanisms and may lead to the identification of novel therapies.

\section{REFERENCES}

I Potter CJ, Huang H, Xu T. Drosophila Tscl functions with Tsc2 to antagonize insulin signaling in regulating cell growth, cell proliferation, and organ size. Cell 200I; 105:357-68. doi:10.1016/ S0092-8674(0I)00333-6

2 Bissler JJ, McCormack FX, Young LR et al. Sirolimus for angiomyolipoma in tuberous sclerosis complex or lymphangioleiomyomatosis. N Engl J Med 2008; 358: I40-5I. doi:I0.I056/NEJMoa063564

3 Ehninger D, Han S, Shilyansky $C$ et al. Reversal of learning deficits in a Tsc2+/- mouse model of tuberous sclerosis. Nat Med 2008; I4:843-8. doi: I0.1038/nm I 788

4 Neptune ER, Frischmeyer PA, Arking DE et al. Dysregulation of TGF-beta activation contributes to pathogenesis in Marfan's syndrome. Nat Genet 2003; 33:407-I I. doi: I0.1038/ng I I I6 
5 Loeys BL, Chen J, Neptune ER et al. A syndrome of altered cardiovascular, craniofacial, neurocognitive and skeletal development caused by mutations in TGFBRI or TGFBR2. Nat Genet 2005; 37:275-81. doi:10.1038/ng15II

6 Habashi JP, Judge DP, Holm TM et al. Losartan, an ATI antagonist, prevents aortic aneurysm in a mouse model of Marfan's syndrome. Science 2006; 3 | 2: | 17-2 I. doi: I0. I | 26/science. I 24287

7 Brooke BS, Habashi JP, Judge DP et al. Angiotensin II blockade and aortic-root dilation in Marfan's syndrome. N Engl J Med 2008; 358:2787-95. doi:10.1056/NEJMoa0706585

8 Davies $\mathrm{H}$, Bignell GR, Cox C et al. Mutations of the BRAF gene in human cancer. Nature 2002; 4/7:949-54. doi:10.1038/ nature00766

9 Patton EE, Widlund HR, Kutok JL et al. BRAF mutations are sufficient to promote nevi formation and cooperate with $\mathrm{p} 53$ in the genesis of melanoma. Curr Biol 2005; 15:249-54. doi:10.1016/j. cub.2005.01.031

10 Smith FJ, Irvine AD, Terron-Kwiatkowski A et al. Loss-of-function mutations in the gene encoding filaggrin cause ichthyosis vulgaris. Nature Genet 2006; 38:337-42. doi:10.1038/ng I743

II Sandilands A, Sutherland C, Irvine AD et al. Filaggrin in the frontline: role in skin barrier function and disease.J Cell Sci 2009; 122:1285-94. doi:10.1242/jcs.033969

12 Palmer CN, Irvine AD, Terron-Kwiatkowski A et al. Common lossof-function variants of the epidermal barrier protein filaggrin are a major predisposing factor for atopic dermatitis. Nature Genet 2006; 38:44I-6. doi: 10.1038/ng I767

13 Van Deutekom JC, Janson AA, Ginjaar IB et al. Local dystrophin restoration with antisense oligonucleotide PRO05I. N Engl J Med 2007; 357:2677-86. doi:I0.1056/NEJMoa073108

14 National Institute for Health and Clinical Excellence. Identification and management of familial hypercholesterolaemia. London: NICE; 2008. Available from: http://www.nice.org.uk/nicemedia/pdf/ CG07INICEGuideline.pdf
15 Umans-Eckenhausen MA, Defesche JC, Sijbrands EJ et al. Review of first 5 years of screening for familial hypercholesterolaemia in the Netherlands. Lancet 200I; 357:165-8. doi:I0.1016/S0I406736(00)03587-X

16 Marks $D$, Wonderling $D$, Thorogood $M$ et al. Cost effectiveness analysis of different approaches of screening for familial hypercholesterolaemia. BMJ 2002: 324:|303. doi:|0.|136/ bmj.324.7349.1303

17 Starr B, Hadfield SG, Hutten BA et al. Development of sensitive and specific age- and gender-specific low-density lipoprotein cholesterol cutoffs for diagnosis of first-degree relatives with familial hypercholesterolaemia in cascade testing. Clin Chem Lab Med 2008; 46:79I-803. doi:I0.I5I5/CCLM.2008.I35

18 Wald DS, Bestwick JP, Wald NJ. Child-parent screening for familial hypercholesterolaemia: screening strategy based on a meta-analysis. BMJ 2007; 335:599-603. doi:I0.I I36/bmj.39300.6I6076.55

19 Kaneda A,Wang CJ, Cheong $R$ et al. Enhanced sensitivity to IGF-II signaling links loss of imprinting of IGF2 to increased cell proliferation and tumor risk. Proc Natl Acad Sci USA 2007; 104:20926-31. doi:I0.1073/pnas.0710359105

20 Maher ER, Brueton LA, Bowdin SC et al. Beckwith-Wiedemann syndrome and assisted reproduction technology (ART). J Med Genet 2003; 40:62-4. doi: I 0.I I36/jmg.40.I.62

21 Wardle J, Carnell S, Haworth CMA et al. Evidence for a strong genetic influence on childhood adiposity despite the force of the obesogenic environment. Am J Clin Nutr 2008; 87:398-404.

22 Willer CJ, Speliotes EK, Loos RJF et al. Six new loci associated with body mass index highlight a neuronal influence on body weight regulation. Nature Genet 2009; 41:25-34. doi:10.1038/ng.287

23 Dina C, Meyre D, Gallina S. Variation in FTO contributes to childhood obesity and severe adult obesity. Nature Genet 2007; 39:724-6. doi: $10.1038 / n g 2048$

24 Wardle J, Carnell S, Haworth CM et al. Obesity associated genetic variation in FTO is associated with diminished satiety. J Clin Endocrinol Metab 2008; 93:3640-3. doi:10.1210/jc.2008-0472

\section{CONTINUING MEDICAL EDUCATION}

\section{Online distance learning and self-assessment from the RCPE}

To allow users to update their general medical knowledge the online CME modules contain a variety of material, including articles, interactive cases and quizzes, webstreamed lectures and self-assessment multiple choice questions (MCQs). Each module also includes material of particular relevance to those undertaking core medical training.

CME modules are eligible for external distance learning CPD credits under the UK Federation of
Royal Colleges of Physicians CPD scheme. To claim these credits you must take the MCQ test for the module. Full instructions are given with each module.

\section{The College's online CME} programme is available to Fellows, Collegiate Members, Associates and e-Associates. Log on to the Fellows' and Members' secure area at: http://www.rcpe.ac.uk. If you have lost or forgotten your username or password please contact: webmaster@rcpe.ac.uk

\section{Currently available for CPD points}

- Psychiatry (September 2009)

- Acute medicine (April 2009)

- Diabetes \& Endocrinology (November 2008)

\section{Coming soon}

- Cardiology

- Palliative care

- Tropical medicine 